\title{
The role of Narrow-band Imaging Cystoscopy in Non-muscular Invasive Bladder Cancer Management
}

\author{
IOAN SCARNECIU1,2*, IO NUT-ALEXANDRU BANUTA2, CAMELIA CORNELIA SCARNECIU1,2, ADRIAN BRINZA2, LAURIAN MAXIM ${ }^{1,2}$, \\ ALEXANDRU GEORGIAN LACULICEANU ${ }^{1,2}$, HODADE DANIEL PORAV ${ }^{3,4}$, MOGA CIPRIAN TODEA ${ }^{3,4}$, VERONICA GHIRCA ${ }^{3,4}$, \\ MARTHA ORSOLYA 3,4 \\ 'University of Transilvania Faculty of medicine, 56th Nicolae Balcescu Str., 500019, Brasov, Romania \\ ${ }^{2}$ Clinical Emergency County Hospital, 25-27th Bucuresti Road, 500326, Brasov, Romania \\ 3University of Medicine, Pharmacy, Sciences and Technology of Targu Mures, 38 Gheorghe Marinescu Str., 540139, Targu Mures, \\ Romania \\ ${ }^{4}$ Clinical Emergency County Hospital, $1{ }^{\text {st }}$ Gheorghe Marinescu Str., 540103, Targu Mures, Romania
}

\begin{abstract}
Cystoscopy is the most common assessment method for the lower urinary tract, with the primary goal of establishing pathology management that can occur at this level. The most used method of performing cystoscopy is with white light, but it can lead to omission of lesions, especially when considering millimetric tumor formations. New light source technologies are under development, such as narrow-band cystoscopy. Overall, we examined a total of 416 patients, known with tumoral lesions, in WLI followed by reassessment in NBI at the same time and transurethral resection of tumor biopsy specimens. In 37.5\% of the cases, NBI highlighted 1 to 3 tumors in addition to WLI. In 178 cases, tumor formations were newly discovered, and in 238 patients these were recurrent. Histopathological diagnosis indicated the pTa stage in $67.3 \%$ of cases, in $27.64 \%$ patients presented with pT1 stage and in $5.04 \%$ of cases CIS was identified. In terms of grading, G1 was identified in 205 of the cases, 124 patients presenting G2 and G3 occurred in 87 of the cases.
\end{abstract}

Keywords: Cystoscopy, NBI, WLI

Bladder carcinoma is the most common neoplasm of the urothelial tract. Approximately $75-85 \%$ of patients with bladder carcinoma are confirmed with non-muscular invasive bladder cancer (NMIBC) that includes mucosa (Ta and CIS) and submucosa (T1) [1, 2]. Smoking is a well-known risk factor for bladder cancer mostly in men, also being a cause for infertility and erectile dysfunction $[3,4]$.

The current diagnostic and therapeutic management for NMIBC is transurethral resection of bladder tumors (TURBT) with white light imaging (WLI). However, small papillary tumors.

or carcinoma in situ (CIS) may easily be missed by WLI [5], and for this cases, new types of imaging are used for the improvement of diagnosis, treatment and a lower rate of recurrence.

A new type of cystoscopy has been used in the last years, using narrow-band imaging (NBI), improving the detection and treatment of NMIBC [6], also decreasing the rate of recurrence, placing narrow-band filters in front of a conventional light source to achieve illumination of tissues of a particular wavelength. This optical technique filters white light into two bands of light in the blue $(415 \mathrm{~nm})$ and green $(540 \mathrm{~nm})$ spectrum, which penetrates tissue only superficially and is strongly absorbed by hemoglobin [7].

The study aimed to determine if NBI can improve the detection of NMIBC and also the management of these cases.

\section{Experimental part}

Materials and methods

We performed a prospective study where we compared the two techniques of imaging (WLI and NBI) on a total of 416 patients with ages between 34 and 80 years old presenting known primary or recurrent tumor. The study was conducted between September 2017 and February 2019, using an Olympus NBI system. The technique consisted of evaluating the bladder with WLI and after performing an NBI reevaluation. In all cases, TURBT with biopsy was performed, and the histopathological result indicated NMIBC.

\section{Results and discussions}

In total, 416 cystoscopies with WLI and NBI were performed, on $185(44.47 \%)$ females and 231 males $(55.52 \%)$.

In 178 cases $(42.78 \%)$ the patients presented primary tumor and 238 of cases (57.21\%) there was recurrent tumors. From the total number of cases with a recurrent tumor, $154(64.70 \%)$ patients presented previous instillations, and in 84 (35.29\%) of cases, there were not reported instillations.

In 182 cases (37.5\%) the NBI detected an additional 1 to 3 new tumors after performing WLI cystoscopy, divided as follows: 1 new tumor in 84 (46.15\%) patients, two tumors in $69(37.91 \%)$ cases and three new tumors in 29 $(15.93 \%)$ patients.

For 84 patients $(35.29 \%)$ there was no prior intravesical instillation, but in 154 cases (64.7\%) the patients had bladder instillation of chemotherapy and for all the NBI evaluation was 2 months after to avoid false positive results (table 1 ).

The histopathological result indicated pTa stage in 280 cases $(67.3 \%)$, in $115(27.64 \%)$ patients presented pT1, and in 21 cases $(5.04 \%)$ CIS was identified.

Also, 205 patients (49.27\%) presented Grading 1, in 124 cases (29.8\%) we identified Grading 2 and Grading 3 was found in 87 patients $(20.91 \%$ ) (table 2 ).

It is critical for the management of NMIBC to identify all tumor lesions by a new diagnostic technique, detecting low-grade and non-invasive bladder cancer, instead of

* email: urologie_scarneciu@yahoo.com 
Table 1

PATIENTSCHARACTERISTICS

\begin{tabular}{|c|c|}
\hline PATIENTS & NUMBER $(\%)^{--}$ \\
\hline & 416 \\
\hline \multicolumn{2}{|l|}{ GENDER } \\
\hline MALE & $231(55.52 \%)$ \\
\hline FEMALE & $185(44.47 \%)$ \\
\hline AGE (AVERAGE) & $34-80$ years $(55.3)$ \\
\hline \multicolumn{2}{|l|}{ BLADDER TUMOR } \\
\hline PRIMARY- & $178(42.78 \%)$ \\
\hline RECURRENT & $238(57.21 \%)$ \\
\hline \multicolumn{2}{|c|}{ PRIOR INSTILLATIONS } \\
\hline $\mathrm{YES}^{-}$ & $154(64.70 \%)$ \\
\hline $\mathrm{NO}^{--\cdot+}$ & $84(35.29 \%)$ \\
\hline \multicolumn{2}{|c|}{ NEW TUMORS IDENTIFIED ON NBI } \\
\hline 1 TUMOR & $84(46.15 \%)$ \\
\hline 2 TUMORS $^{-1}$ & $69(37.91 \%)$ \\
\hline 3 TUMORS & $29(15.93 \%)$ \\
\hline
\end{tabular}

finding them at a later evaluation with a higher grade and staging $[8,9]$, because they have a risk for progression at five years of $17-45 \%$ [9], with invasion of ureteral oriffices and secondary renal failure [10,11] or metastases [12-17].

Beside hematuria, other symptoms can occur in bladder cancer, such as overactive bladder, or urinary retention, which makes the differential diagnosis more difficult [1822]. Complications after cystoscopy can often appear, such as hematuria, urinary tract infections $[23,24]$, pyelonephritis in patients with JJ ureteral stent [25-29], or
Table 2

PATHOLOGICAL RESULTSOF LESIONSIDENTIFIED BY WLI AND NBI

\begin{tabular}{|c|c|}
\hline HISTOPHATOLOGICAL RESULTS & NUMBER (\%) \\
\hline STAGE & \\
\hline pTa & $280(67.3 \%)$ \\
\hline pTl & $115(27.64 \%)$ \\
\hline CIS & $21(5.04 \%)$ \\
\hline GRADING & \\
\hline Gl & $205(49.27 \%)$ \\
\hline G2 & $124(29.8 \%)$ \\
\hline G3 & $87(20.91 \%)$ \\
\hline
\end{tabular}

other complications [30-32]. It is very important to exclude any other causes of hematuria [33-36], such as a prostatic carcinoma in male [37-40] or bladder endometriosis in women [41-44].

The NBI evaluation and TURBT of NMIBC show that it has fewer subsequent recurrences and longer recurrencefree gap than WLI [45]. Any overlooked tumors will be considered as recurrence at a later evaluation. Two articles that compared the treatment effectiveness using NBI and WLI TURBT shows that the 3-months recurrence rate was $15.0 \%$ and $30.5 \%$ [46], also $3.9 \%$ and $16 \%$ [47].

Another study indicates that minimal variations appeared between a novice urologist in NBI and one with more experience, concluding there is no curve in learning $\mathrm{NBI}$ cystoscopy and TURBT [48]. The article of Herr et al., proves that variations on an individual level between urologists using NBI has no impact on the quality of NMIBC management [49-52], but the number of biopsies forms normal mucosa can affect the specificity of the procedure [52-54].
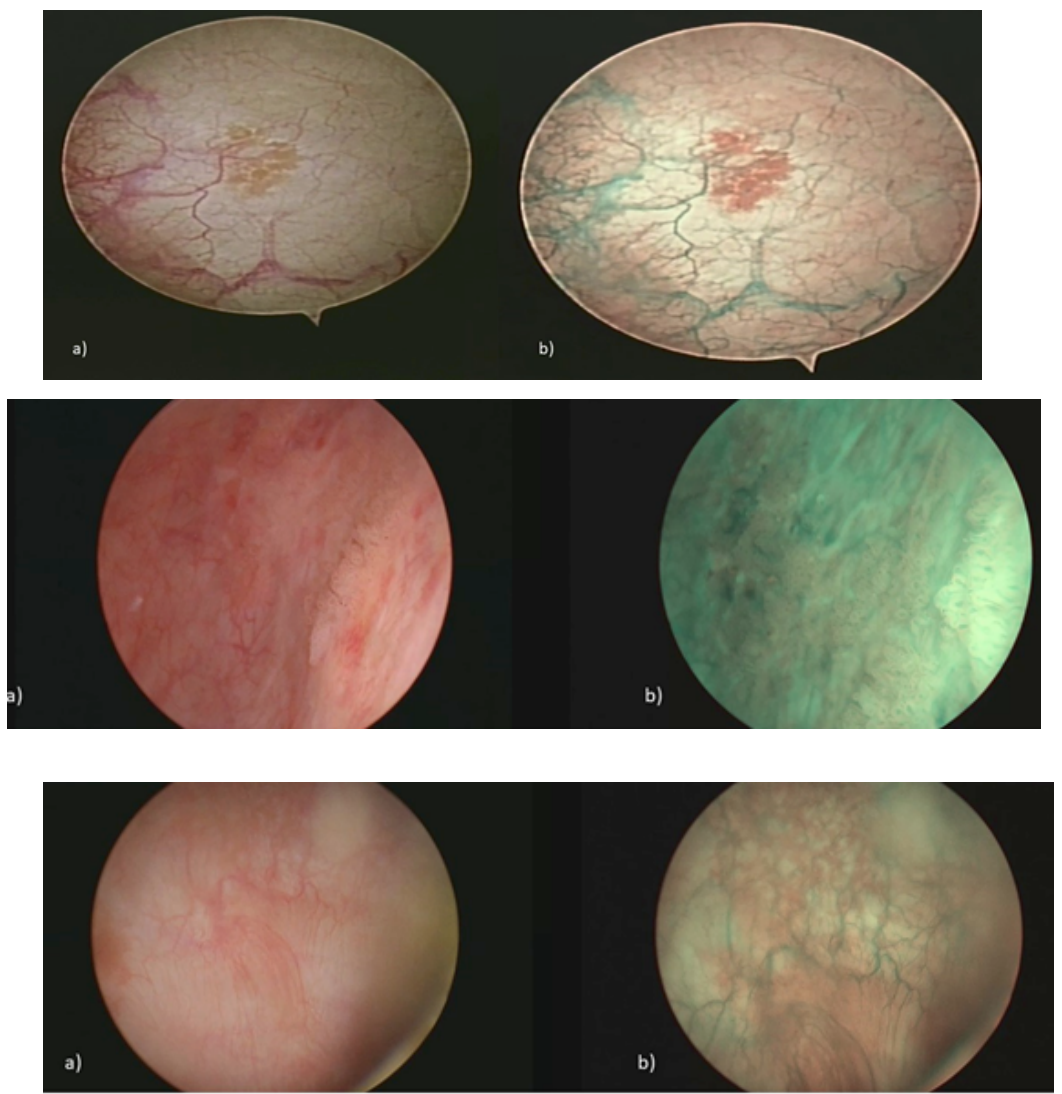

Fig. 1. Neoplasic lesions of the bladder: a) Overlooked tumor in WLI cystoscopy; b) Improved detection by NBI

Fig. 2. Neoplasic lesions of the bladder: a) Incomplete evaluation of tumors in WLI; b) Full aspect of lesions in NBI

Fig. 3. Neoplasic lesions of the bladder: a) WLI image of $\mathrm{CIS}$; b) Enhanced contrast of the same tumors with $\mathrm{NBI}$ 


\section{Conclusions}

This study shows that NBI cystoscopy and TURBT significantly improves the detection of primary and recurrent NMIBC compared to WLI, particularly for Ta and $\mathrm{CIS}$ lesions. Patients diagnosed with bladder tumors, investigated throught WLI cystoscopy, had fewer subsequent recurrences and longer recurrence-free intervals when additional investigation, such as NBI cystoscopy, was performed.

The NBI technique increases the contrast between a normal epithelium and a slightly elevated epithelium. In patients with prior bladder instillations, the NBI evaluation must be performed after a safe period of time, to avoid false positive results given by the modification of blood vessel architecture.

\section{References}

1.BABJUK, M., OOSTERLINCK, W., SYLVESTER, R., Eur. Urol., 59, 2011, p. 997-1008.

2.SOBIN, LH., GOSPODAROWICZ, MK., WITTEKIND, C. (eds) TNM Classification of Malignant Tumors, 7th edn. Wiley-Blackwell, L.H. Sobin, M.K. Gospodarowicz, Ch. Wittekind, New York, 2009, p. 262265.

3.SCARNECIU, I., LUPU, S., SCARNECIU, CC., Procedia Soc Behav Sci, 127, 2014, p. 437-442.

4.DIACONU, C.C., MANEA, M., MARCU, D.R., SOCEA, B., SPINU, A.D., BRATU, O.G. Acta Cardiologica 2019. DOI 10.1080/ 00015385.2019.1590498

5.SANTOS CORTES, J.A., GRAHAN, J., SOLOWAY, MS., Int J Urol, 64, 2011; p. 18-31.

6.HERR, HH., Urol Oncol., 29, 2011, p. 353-357.

7. EAST, JE., SUZUKI, N., STAVRINIDIS. M., Gut, 57, 2008, p. 65-70.8.

8. SOLOWAY, MS., Urol Oncol, 24, 2006, p. 58-61.

9.PALLAG, A., ROSCA, E., TIT, D.M., MUTIU, G., BUNGAU, S.G., POP, O.L., Rom. J. Morphol. Embriol., 56, no. 3, 2015, p. 1103.

10.NICULAE, A., PERIDE, I., MARINESCU-PANINOPOL, A., VRABIE, C.D., GINGHINA, O., JECAN, C.R., BRATU, O.G., Rom J Morphol Embryol, 57, no. 2, 2016, p. 591-594.

11.RADULESCU, D., BALCANGIU STROESCU, A., PRICOP, C., GEAVLETE, B., NEGREI, C., BRATU, O., GINGHINA, O., VACAROIU, I., Rev Chim (Bucharest), 68, no. 1, 2017, p. 52-54.

12.MARCU, R.D., SPINU, A.D., SOCEA, B., BODEAN, M.O., DIACONU, C.C., VASILESCU, F., NEAGU, T.P., BRATU, O.G., Rev Chim (Bucharest), 69, no. 4, 2018, p. 823-830.

13.BRATU, O.G., MARCU, R.D., SOCEA, B., NEAGU, T.P., DIACONU, C.C., SCARNECIU, I., TURCU, F.L., RADAVOI, G.D., BRATILA, E., BERCEANU, C., SPINU, A.D., Rev Chim (Bucharest), 69, no. 7, 2018, p. 1813-1816.

14.CONSTANTINOIU, S., BARLA, R., IOSIF, C., COCIU, L., GINDEA, C., HOARA, P., BRATU, O., RUSITORU, L., Chirurgia (Bucur), 104, no. 3, 2009, p. 363-367.

15.BRATU, O., MISCHIANU, D., SPINU, D., BARLA, R., HOARA, P., CONSTANTINOIU, S., Chirurgia (Bucur), 108, no. 1, 2013, p. 26-31.

16.MARCU, D.R., IONITA-RADU, F., IORGA, L.D., MANEA, M., SOCEA, B., SCARNECIU, I., ISVORANU, G., COSTACHE, R., DIACONU, C.C., BRATU, O.G., Rev Chim (Bucharest), 70, no. 2, 2019, p. 445-448.

17.BRATU, O.G., CHERCIU, A.I., BUMBU, A., LUPU, S., MARCU, D.R., IONITA RADU, F., MANEA, M., FURAU, C., DIACONU, C.C., MISCHIANU, D.L.D., Rev Chim (Bucharest), 70, no. 1, 2019, p. 190-194.

18.SYLVESTER, RJ., VAN DER MEIJDEN, AP., OOSTERLINCK, W., Eur Urol, 49, 2006, p: 466-475.

19.PANDI, E., MAXIM, LS., CRISTIAN, A., HOGEA, MD., DOCHIT, CM., SCARNECIU, CC., SCARNECIU, I., MIRONESCU, A., J. Urol, 13, no. 4, 2016, p: 2797-2799.

20.MOGA, MA., BANCIU, S., DIMIENESCU, O., BAGIU, NF., SCARNECIU, I., J Pak Med Assoc, 65, no. 1, 2015, p: 76-80.

21DIACONU CC, ARSENE D, BALACEANU A, BARTOS D. Romanian J ournal of Morphology and Embryology, 55, no. 3, 2014, p. 973-976.
22.DIACONU, C. Cor et Vasa, 59, 2017, p. e171-e173.

23.RADULESCU, A., MADAN, V., AUNGURENCI, A., BRATU, O., FARCAS, C., DINU, M., MISCHIANU, D., Romanian J ournal of Military Medicine, 118, no. 3, 2015, p. 20-22.

24.SPINU, D., BRATU, O., POPESCU, R., MARCU, D., RADULESCU, A., MISCHIANU, D., Romanian Journal of Military Medicine, 118, no. 3, 2015, p. 12-15.

25.SCARNECIU, I., LUPU, S., SCARNECIU, CC., COCUZ, ME., SCARNECIU, V., Metal Int, 16, no. 9, 2011, p. 43-45.

26.BUMBU, A., NACER, K., BRATU, O., BERECHET, M., BUMBU, G., BUMBU, B., Proceedings: 14th National Congress of Urogynecology and The National Conference of the Romanian Association for the Study of Pain, Eforie, Romania, 2017, p. 82.

27.BUMBU, G.A., BERECHET, M.C., NACER, K., BUMBU, G., MAGHIAR, O.A., BRATU, O.G., VICAS, R.M., TICA, O., BUMBU, B.A., Romanian Journal of Morphology and Embriology, 59, no. 4, 2018, p. 1173-1177. 28.SCARNECIU, I., BRATU, O.G., COBELSCHI, C.P., NECULOIU, C.D., SCARNECIU, C.C., LUPU, S., BRINZA, A., MARCU, D., SOCEA, B., MAXIM, L., Rev Chim (Bucharest), 69, no. 12, 2018, p. 3406-3409.

29.SPINU, A.D., MARCU, R.D., SOCEA, B., DIACONU, C.C., SCARNECIU, I., SCARNECIU, C., BODEAN, O.M., DRAGOMIRESCU, R.I.F., STANESCU, A.M.A., MISCHIANU, D.L.D., BRATU, O.G., Rev Chim (Bucharest), 69, no. 8,2018 , p. 2061-2063.

30.DIACONU, C.C., DEDIU, G.N., IANCU, M.A. Acta Cardiologica, 73, no. 6,2018 , p. 511-517.

31.TIGLIS, M., NEAGU, T.P., ELFARA, M., DIACONU, C.C., BRATU, O.G., VACAROIU, I.A., GRINTESCU, I.M. Rev Chim (Bucharest), 69, no. 10, 2018, p. 2877-2880.

32.GAMAN, M.A., DOBRICA, E.C., PASCU, E.G., COZMA, M.A., EPINGEAC, M.E., GAMAN, A.M., PANTEA STOIAN, A., BRATU, O.G., DIACONU, C.C. J ournal of Mind and Medical Sciences, 69, no. 1, 2019, p. 157-161.

33.TATARU, A.L., FURAU, G., AFILON, J., IONESCU, C., DIMITRIU, M., BRATU, O.G., TIT, D.M., BUNGAU, S., FURAU, C., J. Clin. Med., 8, no. 1, 2019, E96; https://doi.org/10.3390/jcm8010096.

34.MARCU, R.D., SPINU, A.D., MISCHIANU, D., OPREA, I.S., DIACONU, C., SOCEA, B., BRATU, O.G., Farmacia, 67, no. 1, 2019, p. 50-55.

35.TICA, O.A., TICA, O., ANTAL, L., HATOS, A., POPESCU, M.I., PANTEA STOIAN, A., BRATU, O.G., GAMAN, M.A., PITURU, S.M., DIACONU, C.C. Farmacia, 66, no. 6, 2018, p. 972-976.

36.LASLO, C., PANTEA STOIAN, A., SOCEA, B., PADURARU, D., BODEAN, O., SOCEA, L., NEAGU, T.P., STANESCU, A.M.A., MARCU, D., DIACONU, C. Journal of Mind and Medical Sciences, 5, no. 2, 2018, p. 195-201.

37.DRAGOS, M., SPINU, D., MISCHIANU, D., SOCEA, B., OPREA, I., BRATU, O., Romanian J ournal of Military Medicine, 120, no. 3, 2017, p. 39-42.

38.CIUCA, G.A., BRATU, O., SPINU, D., DINU, M., FARCAS, C., RADULESCU, A., POPESCU, R., MARCU, D., MISCHIANU, D., ARMEAN, P. Romanian Journal of Military Medicine, 119, no. 2, 2016, p. 12-16. 39.POPESCU, R., BRATU, O., SPINU, D., MARCU, D., FARCAS, C., DINU, M., MISCHIANU, D. Romanian J ournal of Military Medicine, 118, no. 3, 2015, p. 16-19.

40.BRATU, O., SPINU, D., OPREA, I., POPESCU, R., MARCU, D., FARCAS, C., DINU, M., MISCHIANU, D., Romanian J ournal of Military Medicine, 118, no. 3, 2015, p. 23-25.

41.STANIMIR, M., CHIUTU, L.C., WESE, S., MILULESCU, A., NEMES, R.N., BRATU, O., Rom J Morphol Embryol, 57, no. 2 Suppl, 2016, p. 849-852.

42.MEHEDINTU, C., ANTONOVICI, M., BRINDUSE, L., BRATILA, E., STANCULESCU, R., BERCEANU, C., BRATU, O., PITURU, S., ONOFRIESCU, M., MATASARIU, D.R., Rev Chim (Bucharest), 69, no. 3, 2018, p. 581-584.

43.SOCEA, L.I., VISAN, D.C., BARBUCEANU, S.F., APOSTOL, T.V., BRATU, O.G., SOCEA, B., Rev Chim (Bucharest), 69, no. 4, 2018, p. 795-797.

44.BODEAN, O., BRATU, O., BOHILTEA, R., MUNTEANU, O., MARCU, D., SPINU, D.A., VACAROIU, I.A., SOCEA, B., DIACONU, C.C., FOMETESCU GRADINARU, D., CIRSTOIU, M., Rev Chim (Bucharest), 69, no. 6, 2018, p. 1411-1415. 
45.PRICOP, C., DRAGOMIR, S., MARDARI, B., BARDAN, R., SCARNECIU, I., ORSOLYA, M., Biol. Sci., 66, no. 4, 2014, p. 1581-1584.

46.HERR, HW., DONAT, SM., BJU Int., 107, no. 3, 2011, p. 396-8.

47.CAUBERG, E.C., MAMOULAKIS, C., DE LA ROSETTE, J .J ., DE REIJ KE, T.M., World J. Urol., 29, 2011, p. 503.

48.NASELLI, A., INTROINI, C., TIMOSSI, L., et al., Eur. Urol, 61, 2012, p. 908-13.

49. KAUSCH, I., SOMMERAUER, M., MONTORSI, F., Eur. Urol., 57, 2010, p. 595-606.

50. SHEN, Y.J ., ZHU, Y.P., YE, D.W., Int. Urol. Nephrol, 44, 2012, p. 4517.
51.TATSUGAMI, K., KUROIWA, K., KAMOTO, T., J. Endourol, 24, 2010, p. 1807-11.

52.HERR, H., DONAT, M., DALBAGNI, G., TAYLOR, J., BJU Int., 106, 2010, p. 53-5.

53.ABDEL-DAIM, M.M., AHMED, A., IJ AZ, J., ABUSHOUK, A.I., AHMED, H., NEGIDA, A., et al., Environ. Sci. Pollut. Res., 26, no. 8, 2019, p. 8080.

54.ABDEL-DAIM, M.M., ABO-EL-SOOUD, K., ALEYA, L., BUNGAU, S.G., NAJDA, A., SALUJA, R., Oxid. Med. Cell. Longev., 2018, ID 6276438, 2018 https://doi.org/10.1155/2018/6276438.

Manuscript received: 15.09 .2018 